\title{
Editorial
}

\section{Some Recent Developments in Applied Functional Analysis}

\author{
Manuel Ruiz Galán, ${ }^{1}$ María Isabel Berenguer, ${ }^{1}$ Gabriel N. Gatica, ${ }^{2}$ and Davide La Torre ${ }^{3}$ \\ ${ }^{1}$ Departamento de Matemática Aplicada, E.T.S. Ingeniería de Edificación, Universidad de Granada, 18071 Granada, Spain \\ ${ }^{2}$ Departamento de Ingeniería Matemática, Universidad de Concepción, Casilla 160-C, Concepción, Chile \\ ${ }^{3}$ Department of Economics, Business and Statistics, University of Milan, 20122 Milan, Italy
}

Correspondence should be addressed to Manuel Ruiz Galán; mruizg@ugr.es

Received 21 February 2013; Accepted 21 February 2013

Copyright (C) 2013 Manuel Ruiz Galán et al. This is an open access article distributed under the Creative Commons Attribution License, which permits unrestricted use, distribution, and reproduction in any medium, provided the original work is properly cited.

From its early stages, the intensive development of functional analysis and the remarkable advances of its methods cannot be explained without its link with other areas of mathematics and, above all, its role as an essential framework for numerical analysis and computer simulation, PDEs, modeling realworld phenomena, variational inequalities, or optimization, just to name a few.

In this special issue we highlight some aspects of functional analysis which are used in connection with other branches of mathematics or science, either as a direct application or as a theoretical result which is essential for such an application.

Although it is not possible to collect here the huge production of the research activity on this vast field of modern mathematics, the selected works gather together a range of topics which reflect some of the current research on applied functional analysis: bases in Banach spaces, wavelet transforms, fixed point theory, and applications to ODEs, electronic circuit simulation, or numerical solution of PDEs, integral equations, or problems on option pricing in mathematical finance. In this way, we have achieved one of our purposes, which is the exchange of ideas among researchers working both in abstract and applied functional analysis.

\section{Acknowledgments}

We would like to express our sincere thanks to the authors for contributing to this issue, as well as to the anonymous reviewers for their generous and accurate refereeing process and their valuable comments and suggestions. Without their evident support this special issue would not have come out. We want also acknowledge with gratitude the assistance and help provided by the editorial board members of this Journal during the preparation of this special issue.

Manuel Ruiz Galán María Isabel Berenguer Gabriel N. Gatica Davide La Torre 


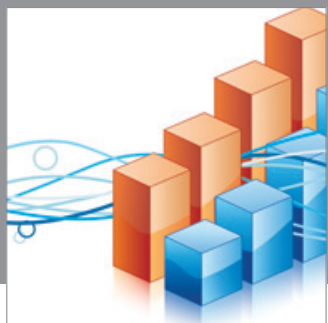

Advances in

Operations Research

mansans

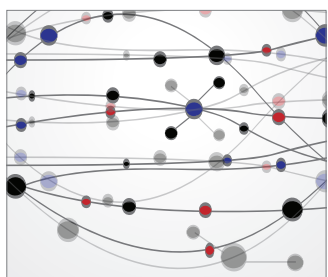

The Scientific World Journal
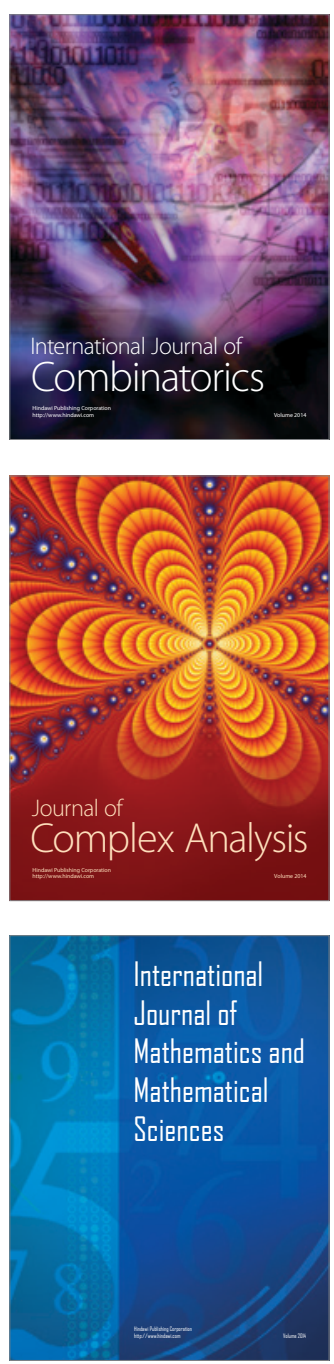
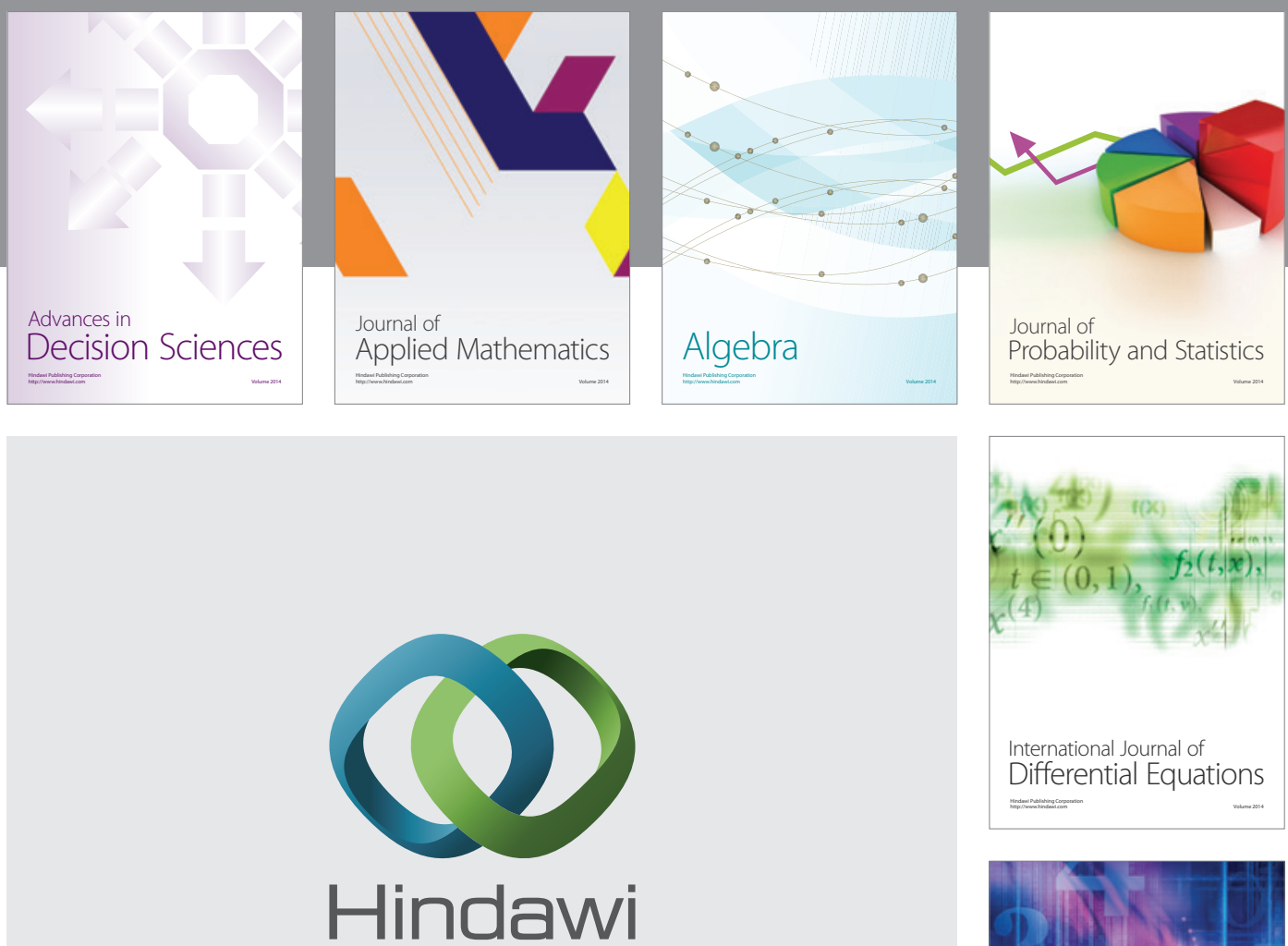

Submit your manuscripts at http://www.hindawi.com
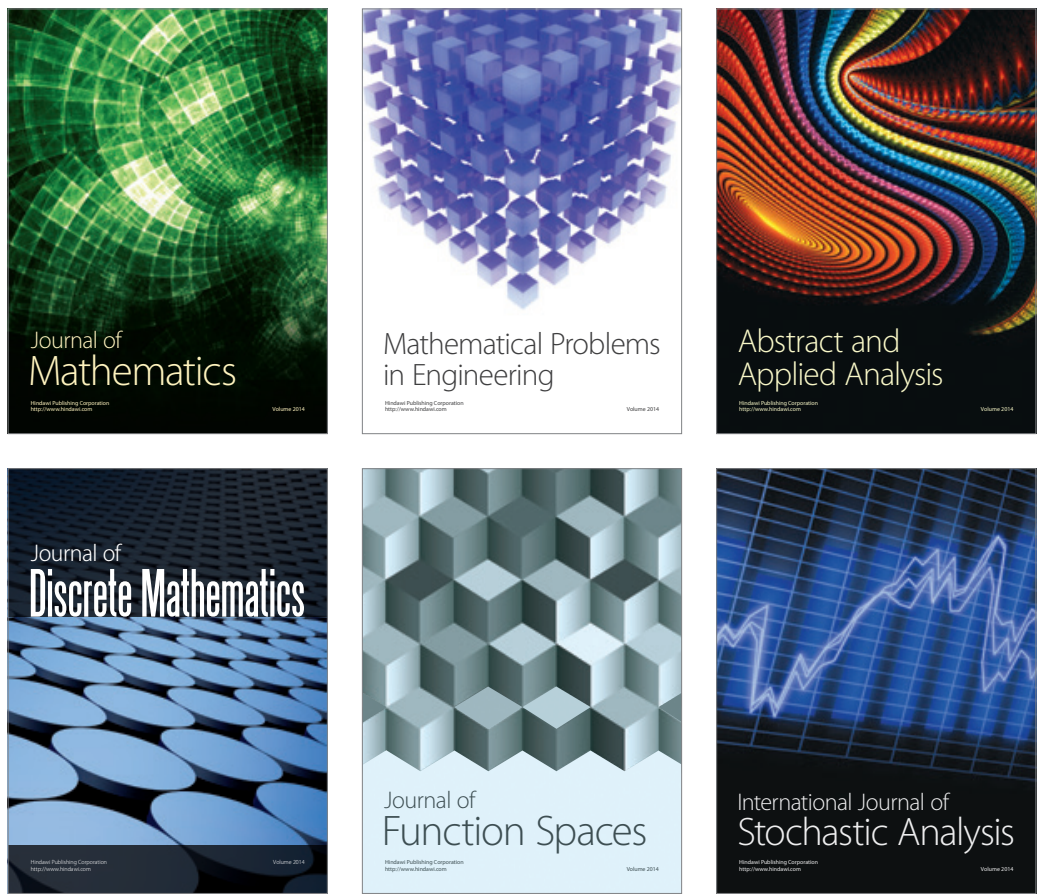

Journal of

Function Spaces

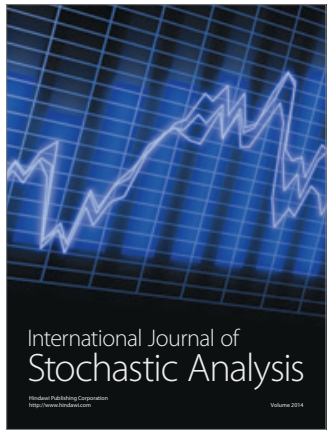

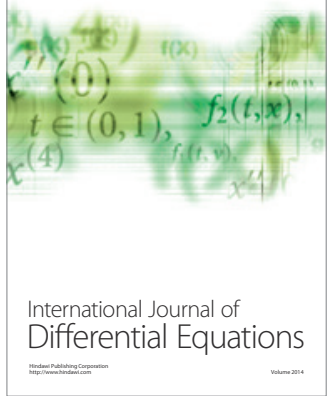
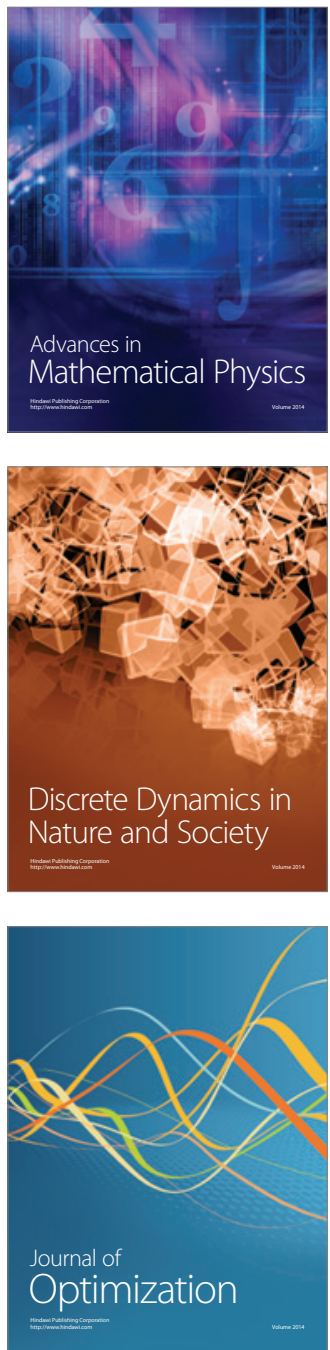